\title{
An example of Crepant Resolution Conjecture in two steps
}

\author{
Renzo Cavalieri and Gueorgui Todorov
}

\begin{abstract}
We study the relation among the genus 0 Gromov-Witten theories of the three spaces $\mathcal{X} \leftarrow \mathcal{Z} \leftarrow Y$, where $\mathcal{X}=\left[\mathbb{C}^{2} / \mathbb{Z}_{3}\right], \mathcal{Z}$ is obtained by a weighted blow-up at the stacky point of $\mathcal{X}$, and $Y$ is the crepant resolution of the $A_{2}$ singularity. We formulate and verify a statement generalizing the Crepant Resolution Conjecture of Bryan and Graber, that we call the Crepant Partial Resolution Conjecture.
\end{abstract}

\section{Introduction}

The Crepant Resolution Conjecture (CRC), loosely stating that a Gorenstein orbifold $\mathcal{X}$ and a crepant resolution $Y$ have "equivalent" Gromov-Witten theories, has been at the center of attention in the past few years.

This conjecture was originally formulated in physics by Zaslow [16] and Vafa [15], and formulated in a mathematically rigorous way by Ruan in [14]. Bryan et al. [3] proposed a stronger formulation, that however holds under the technical assumption of $\mathcal{X}$ being hard Lefschetz (see [3, page 2]). Subsequent work of Coates et al. $[6,7]$ gave rise to a more general statement (written in [8]) in terms of Givental formalism. Several instances of this conjecture have been verified, and a proof of the conjecture for all toric orbifolds was recently announced by Iritani.

This work stems from a somewhat orthogonal question: does the CRC factor? More precisely, given a chain of crepant partial resolutions

$$
\mathcal{X} \leftarrow \mathcal{Z}_{1} \leftarrow \cdots \leftarrow \mathcal{Z}_{r} \leftarrow Y,
$$

is it possible (and meaningful) to obtain partial crepant resolution statements at each step, and the usual CRC as a composition?

We analyze in detail the first non-trivial example of this situation. For the hard Lefschetz orbifold $\mathcal{X}=\left[\mathbb{C}^{2} / \mathbb{Z}_{3}\right]$, with coarse moduli space the $A_{2}$ surface singularity, the $\mathrm{CRC}$ is proven in the strongest possible form in [2]: a 
linear change of variable between the cohomologies and specialization after analytic continuation of the quantum parameters match the Gromov-Witten potentials.

A weighted blow-up at the origin of $\mathcal{X}=\left[\mathbb{C}^{2} / \mathbb{Z}_{3}\right]$ produces the total space of the canonical bundle of a weighted projective line with weights 1 and 2 , which we denote by $\mathcal{Z}$.

The main results of this paper are formulating a statement for the crepant partial resolution conjecture (CPRC, stated in detail in Section 1) and verifying this statement for the above geometry. More precisely,

Theorem 2.1. We express in closed form the genus 0 (extended) GromovWitten potential $\hat{\mathcal{F}}_{\mathcal{Z}}$ of $\mathcal{Z}$.

The potential is computed using Atyiah-Bott localization to reduce to hyperelliptic Hodge integral computations from [4]. By imposing the preservation of the Poincare' pairing we produce the change of variables relating the potentials of $Y$ and $\mathcal{Z}$. The extended potential (introduced in [3, Section 2.2]) is used to invert such transformation. Finally by composing the changes of variable, we obtain:

Theorem 3.2. We exhibit linear changes of variables and specializations of the quantum parameters that match, after analytic continuation, $\hat{\mathcal{F}}_{Y}$ with $\hat{\mathcal{F}}_{\mathcal{Z}}$ and $\hat{\mathcal{F}}_{\mathcal{Z}}$ with $\hat{\mathcal{F}}_{\mathcal{X}}$, thus verifying the CPRC.

\section{Background and statement of CPRC}

In this section, we recall the extended Gromov-Witten potential and the Bryan-Graber statement of the CRC. We follow the general notation introduced in [3], and specifically the notation in [2, Section 1.1], for the potentials of $\left[\mathbb{C}^{2} / \mathbb{Z}_{3}\right]$ and its resolution.

For an orbifold $\mathcal{X}$, an element $\widehat{\beta}$ of the orbifold Neron-Severi group $\widehat{N S}_{1}(\mathcal{X})$ consists of a curve class $\beta=\sum_{1}^{r} \beta_{i} C_{i}$ in $\mathcal{X}$ and a collection of integers $\left\{\hat{\beta}_{1}, \ldots, \hat{\beta}_{s}\right\}$, one for each age one component of the inertia stack of $\mathcal{X}$. The class $\widehat{\beta}$ is called effective if the curve is effective and the integers are non-negative. For an effective class $\widehat{\beta}$ the moduli space $\bar{M}_{g, n}(\mathcal{X}, \widehat{\beta})$ is parameterizing genus $g$ twisted stable maps to $\mathcal{X}$ with degree $\beta, n$-ordered marked points and $\hat{\beta}_{i}$ unordered twisted points mapping to the $i$ th component of 
the inertia stack. The extended potential is defined:

$$
\begin{aligned}
\widehat{F}^{\mathcal{X}} & \left(x_{0}, \ldots, x_{a}, q_{1}, \ldots, q_{r}, u_{1}, \ldots, u_{s}\right) \\
& =\sum_{n_{0}, \ldots, n_{a}=0}^{\infty} \sum_{\widehat{\beta}}\left\langle\delta_{0}^{n_{0}} \cdots \delta_{a}^{n_{a}}\right\rangle_{\widehat{\beta}} \frac{x_{0}^{n_{0}}}{n_{0} !} \cdots \frac{x_{a}^{n_{a}}}{n_{a} !} u_{1}^{\hat{\beta}_{1}} \cdots u_{s}^{\hat{\beta}_{s}} q_{1}^{\beta_{1}} \cdots q_{r}^{\beta_{r}} .
\end{aligned}
$$

If $\delta_{1}, \ldots, \delta_{s}$ are a basis for the age one twisted sectors, then:

$$
\left\langle\delta_{0}^{n_{0}} \cdots \delta_{a}^{n_{a}}\right\rangle_{\widehat{\beta}}^{\mathcal{X}}=\frac{1}{\prod_{1}^{s} \hat{\beta}_{i} !}\left\langle\delta_{0}^{n_{0}}, \delta_{1}^{n_{1}+\hat{\beta}_{1}}, \ldots, \delta_{s}^{n_{s}+\hat{\beta}_{s}}, \ldots, \delta_{a}^{n_{a}}\right\rangle_{\beta}^{\mathcal{X}},
$$

i.e., the extended potential is related to the ordinary potential by;

$$
\widehat{F}^{\mathcal{X}}=F^{\mathcal{X}}\left(x_{0},\left(x_{1}+u_{1}\right), \ldots,\left(x_{s}+u_{s}\right), x_{s+1}, \ldots, x_{a}, q_{1}, \ldots, q_{r}\right) \text {. }
$$

Next, we formulate the CPRC.

Crepant Partial Resolution Conjecture. Let $\pi: \mathcal{Z} \rightarrow \mathcal{X}$ be a crepant map of Gorenstein orbifolds satisfying the hard Lefschetz condition (see [3, Definition 1.1]). Assume $\mathcal{Z}$ has $r+s$ curve classes, $s$ of which are contracted by the map to $\mathcal{X}$. Denote by $S$ the set (in an appropriate positive basis of the $H_{2}(\mathcal{X})$ ) of contracted curve classes, and by $R$ the set of curve classes that map birationally onto their image.

There exists a graded linear isomorphism

$$
L: H^{*}(\mathcal{Z}) \rightarrow H_{\text {orb }}^{*}(\mathcal{X})
$$

and roots of unity $c_{1}, \ldots, c_{r+s}$ such that the following conditions hold.

1. The inverse of $L$ extends the map $\pi^{*}: H^{*}(\mathcal{X}) \rightarrow H^{*}(\mathcal{Z})$.

2. Regarding the potential function $\widehat{F^{Z}}$ as a power series in $z_{0}, \ldots, z_{a}$, $q_{1}, \ldots, q_{r+s}$, the coefficients admit analytic continuations from $\left(q_{1}, \ldots\right.$, $\left.q_{s}\right)=(0, \ldots, 0)$ to $\left(q_{1}, \ldots, q_{s}\right)=\left(c_{1}, \ldots, c_{s}\right)$.

3. The potential functions $\widehat{F}^{\mathcal{X}}$ and $\widehat{F}^{\mathcal{Z}}$ are equal after the substitution

$$
z_{i}=\sum_{j} L_{i}^{j} x_{j}, \quad q_{i}= \begin{cases}c_{i} e^{\sum_{j} L_{i}^{j} u_{j}}, & \text { when } i \in S, \\ c_{i} q_{i}, & \text { when } i \in R .\end{cases}
$$

The CPRC naturally recovers the Bryan-Graber version of the CRC when the map is an actual crepant resolution of the singularities of $\mathcal{X}$. 
Let $\mathcal{X}=\left[\mathbb{C}^{2} / \mathbb{Z}_{3}\right]$, where $\mathbb{Z}_{3}$ acts by opposite weights and $Y$ the crepant resolution of the coarse moduli space $\mathbb{C}^{2} / \mathbb{Z}_{3}$. In $[2]$, Bryan et al. give a closed form expression for the equivariant genus 0 Gromov-Witten potential of $\mathcal{X}$ (Theorem 1.2), of $Y$ (Theorem 1.1), and verify the CRC for this pair (Theorem 1.3).

In particular, they exhibit an explicit change of variables that identifies the potentials. Here, we tweak this change of variable to the extended potential case:

$$
\begin{array}{rlrl}
y_{0} & =x_{0}, \\
y_{1} & =\frac{i}{\sqrt{3}}\left(\omega x_{1}+\bar{\omega} x_{2}\right), & & q_{1}=\omega \mathrm{e}^{\frac{i}{\sqrt{3}}\left(\omega u_{1}+\bar{\omega} u_{2}\right)}, \\
y_{2} & =\frac{i}{\sqrt{3}}\left(\bar{\omega} x_{1}+\omega x_{2}\right), & & q_{2}=\omega \mathrm{e}^{\frac{i}{\sqrt{3}}\left(\bar{\omega} u_{1}+\omega u_{2}\right)} .
\end{array}
$$

\section{The potential}

\subsection{Set-up and notation}

Our main computational technique is virtual localization, for which we refer to [10], and moduli spaces of admissible covers, for which we refer to [5]. Let $\mathcal{Z}$ be the weighted blow-up of the origin in $\left[\mathbb{C}^{2} / \mathbb{Z}_{3}\right]$ with weights one and two. We consider $\mathcal{Z}$ with the induced orbifold structure. A detailed explanation how this is done in general is in [12]. The stack $\mathcal{Z}$ is isomorphic to a local $\mathbb{P}(1,2)$ : the total space of the canonical line bundle on the weighted projective line. Let us fix some notation. Let $x_{0}$ and $x_{1}$ be homogeneous coordinates on $\mathbb{P}(1,2)$, so that

$$
\left(x_{0}: x_{1}\right)=\left(x_{0} x_{1}^{-1 / 2}: 1\right)=\left(1: x_{1} x_{0}^{-2}\right)
$$

Let $X_{0}=x_{0} x_{1}^{-1 / 2}$ denote the pseudo-coordinate on the chart around 0 , isomorphic to $\left[\mathbb{C} / \mathbb{Z}_{2}\right]$. Let $X_{1}=x_{1} x_{0}^{-2}$ be the coordinate on the chart around $\infty$, isomorphic to $\mathbb{C}$. We have the relation $X_{0}^{2}=X_{1}^{-1}$. The only stacky point of $\mathcal{Z}$ is at $X_{0}=0$ and it has stabilizer group $\mathbb{Z}_{2}$. When we consider moduli spaces of twisted stable maps to $\mathcal{Z}$, the only possible non-trivial stabilizer at points on the source curves is $\mathbb{Z}_{2}$. Therefore from now on, by "stacky point" we mean a point with a $\mathbb{Z}_{2}$ stabilizer.

We call $\mathcal{O}_{\mathbb{P}(1,2)}(1 / 2)$ the positive generator of $\operatorname{Pic} \mathbb{P}(1,2) \cong \mathbb{Z}$. We denote by 1 and $H$ the fundamental class and the class of a point in the untwisted 
sector of $\mathcal{Z}$, by $S$ the fundamental class of the twisted sector. We note that $H$ and $S$ span $H_{C R}^{2}(\mathcal{Z})$. The dual variables to $1, H, S$ are denoted $z_{0}, z_{1}, z_{2}$.

By $\bar{M}_{0, n_{1}, n_{2}}(X, \beta)$ we denote the components of $\bar{M}_{0, n_{1}+n_{2}}(X, \beta)$ on which $n_{2}$ of the evaluation maps go the twisted sector, that is twisted stable maps with $n_{1}+n_{2}$ marked points, $n_{2}$ of which are stacky.

The torus action $T=\mathbb{C}^{*} \times \mathbb{C}^{*}$ on $\mathbb{C}^{2}$ with weights $\left(t_{1}, t_{2}\right)$ induces a natural action on $\mathcal{Z}$. The fixed points for the $T$ action on $\mathcal{Z}$ are 0 and $\infty$. The weights on the base and fiber directions are:

$$
\left(t_{2}-t_{1} / 2,3 t_{1} / 2\right) \quad \text { and } \quad\left(t_{1}-2 t_{2}, 3 t_{2}\right)
$$

\subsection{Degree 0 invariants}

Except for the case with only stacky insertions the degree zero invariants are given by the triple intersections in equivariant cohomology

$$
\langle a, b, c\rangle_{0}=\int_{X} a \cup b \cup c,
$$

which are computed by localization:

$$
\begin{gathered}
\langle 1,1,1\rangle_{0}=\frac{1}{3 t_{1} t_{2}} \quad\langle 1,1, H\rangle_{0}=0=\frac{1}{2} \frac{-t_{1}}{\frac{3 t_{1}}{2}\left(t_{2}-\frac{t_{1}}{2}\right)}+\frac{-2 t_{2}}{3 t_{2}\left(2 t_{1}-2 t_{2}\right)}, \\
\langle 1, H, H\rangle_{0}=\frac{1}{2} \frac{\left(-t_{1}\right)^{2}}{\frac{3 t_{1}}{2}\left(t_{2}-\frac{t_{1}}{2}\right)}+\frac{\left(-2 t_{2}\right)^{2}}{3 t_{2}\left(2 t_{1}-2 t_{2}\right)}=-\frac{2}{3} \\
\langle H, H, H\rangle_{0}=-\frac{2\left(t_{1}+2 t_{2}\right)}{3}, \quad\langle 1, S, S\rangle_{0}=\frac{1}{2}, \quad\langle H, S, S\rangle_{0}=\frac{-t_{1}}{2} .
\end{gathered}
$$

The most interesting degree zero invariants are given by $n$-stacky insertions. The corresponding components of the moduli space parameterize maps that contract the curve to 0. By monodromy considerations (or orbifold Riemann-Roch) the number of insertions $n$ is even (and in particular, $n \geq 4$ ). The invariants are computed over the component of $\bar{M}_{0, n}\left(B \mathbb{Z}_{2}\right)$ where all the evaluation maps go to the twisted sector, which we identify with the moduli space of admissible hyperelliptic covers of a rational curve (here $n=2 g+2$ ):

$$
\left\langle S^{2 g+2}\right\rangle_{0}=\int_{A d m_{g \rightarrow 0,\left(t_{1}, \ldots, t_{2 g+2}\right)}} \mathrm{e}^{e q}\left(R^{1} \pi_{*} f^{*}(L \oplus L)\right) .
$$

The obstruction bundle on admissible covers is obtained by pull-pushing the normal bundle to 0 (isomorphic to two copies of the non-trivial bundle $L$ 
on $B \mathbb{Z}_{2}$, linearized with weights $\frac{3 t_{1}}{2}$ and $\left.t_{2}-\frac{t_{1}}{2}\right)$ via the universal map and universal curve for $\bar{M}_{0, n}\left(B \mathbb{Z}_{2}\right)$. The bundle $R^{1} \pi_{*} f^{*} L$ is isomorphic to the dual of the Hodge bundle pulled back from the natural forgetful morphism $A d m_{g \stackrel{2}{\rightarrow} 0,\left(t_{1}, \ldots, t_{2 g+2}\right)} \rightarrow \overline{\mathcal{M}}_{g}$. We conclude that

$$
\left\langle S^{n}\right\rangle_{0}=-\left(t_{1}+t_{2}\right) \int_{A d m_{g \stackrel{d}{\rightarrow} 0,\left(t_{1}, \ldots, t_{2 g+2}\right)}} \lambda_{g} \lambda_{g-1} .
$$

These integrals have been computed in [9, Corollary 2] or [1].

\subsection{Positive degree}

Let $E \cong \mathbb{P}(1,2)$ be the zero section in $\mathcal{Z}$. The image of any non-constant map must lie in $E$ and so we have that $\bar{M}_{0, n_{1}, n_{2}}(X, d[E]) \cong \bar{M}_{0, n_{1}, n_{2}}(\mathbb{P}(1,2), d)$. With this identification we have that:

$$
\begin{gathered}
{\left[\bar{M}_{0, n_{1}, n_{2}}(X, d[E])\right]^{\mathrm{vir}}=e\left(R^{\bullet} \pi_{*} f^{*} N_{E / X}\right) \text {. The Euler sequence on } \mathbb{P}(1,2)} \\
0 \longrightarrow \mathcal{O}_{\mathbb{P}(1,2)}(-3 / 2) \longrightarrow \mathcal{O}_{\mathbb{P}(1,2)}(-1) \oplus \mathcal{O}_{\mathbb{P}(1,2)}(-1 / 2) \longrightarrow \mathcal{O}_{\mathbb{P}(1,2)} \longrightarrow 0
\end{gathered}
$$

gives the relation

$$
e\left(R^{1} \pi_{*} f^{*} N_{E / X}\right)=\left(t_{1}+t_{2}\right) e\left(R^{1} \pi_{*} f^{*}\left(\mathcal{O}_{\mathbb{P}(1,2)}(-1) \oplus \mathcal{O}_{\mathbb{P}(1,2)}(-1 / 2)\right)\right)
$$

Hence, we can express invariants as:

$$
\begin{aligned}
\left\langle H^{n_{1}} S^{n_{2}}\right\rangle_{d}= & \left(t_{1}+t_{2}\right) \int_{\bar{M}_{0, n_{1}, n_{2}}(\mathbb{P}(1,2), d)} \mathrm{e}\left(R ^ { \bullet } \pi _ { * } f ^ { * } \left(\mathcal{O}_{\mathbb{P}(1,2)}(-1)\right.\right. \\
& \left.\left.\oplus \mathcal{O}_{\mathbb{P}(1,2)}(-1 / 2)\right)\right)
\end{aligned}
$$

The advantage of this reduction is that now we are in the local Calabi-Yau case. Because of the divisor equation it is enough to compute the invariants $\left\langle S^{n}\right\rangle_{d}$, given by the integral:

$$
I_{d, n}:=\int_{\bar{M}_{0,0, n}(\mathbb{P}(1,2), d)} \mathrm{e}\left(R^{\bullet} \pi_{*} f^{*}\left(\mathcal{O}_{\mathbb{P}(1,2)}(-1) \oplus \mathcal{O}_{\mathbb{P}(1,2)}(-1 / 2)\right)\right) .
$$

Consider an auxiliary $\mathbb{C}^{*}$ action on $\mathbb{P}(1,2)$, inherited from the standard action on the coarse moduli space. In homogeneous coordinates:

$$
s \cdot\left(x_{0}, x_{1}\right) \longrightarrow\left(s^{1 / 2} x_{0}, x_{1}\right) .
$$


Table 1: Weights of the lifting of the torus action.

\begin{tabular}{lcc}
\hline & Weight over 0 & Weight over $\infty$ \\
\hline \hline $\mathcal{O}_{\mathbb{P}(1,2)}(-1)$ & 0 & 1 \\
$\mathcal{O}_{\mathbb{P}(1,2)}(-1 / 2)$ & $-1 / 2$ & 0 \\
$T_{\mathbb{P}(1,2)}$ & $+1 / 2$ & 0 \\
\hline
\end{tabular}

The tangent bundle is canonically linearized with weights $1 / 2$ over 0 and -1 over $\infty$. Table 1 shows the choice of the lifting of the action to the bundles $\mathcal{O}_{\mathbb{P}(1,2)}(-1)$ and $\mathcal{O}_{\mathbb{P}(1,2)}(-1 / 2)$ that we make in order to eliminate many fixed loci.

A fixed map for the torus action consists of a collection of genus zero (orbi-)curves mapping of positive degree to $\mathbb{P}(1,2)$ and fully ramified over 0 and $\infty$, attached to other genus zero curves contracting to the two fixed points. The marked points must lie on curves contracted over 0. Our choice of weights forces the possibly contributing localization graphs to have valence one over both 0 and $\infty$. The only fixed locus that survives parameterizes maps $f: \mathcal{C} \longrightarrow \mathbb{P}(1,2)$, where $\mathcal{C}$ has two components $F$ and $C$. The curve $F$ carries all the marked points and it is contracted over zero, whereas $f_{\mid C}$ is a degree $d$ map fully ramified over 0 and $\infty$.

\subsection{Odd degree}

When the degree is odd the node is stacky. Since we must have an even number of stacky points on $\mathcal{C}$, we can only have invariants with an odd number of insertions. The non-contracting curve $C$ is again a weighted projective line with weights one and two. If we choose local coordinates $Y_{0}$ and $Y_{1}$ the map is given by $f^{*} X_{0}=Y_{0}^{d}$. The weight of $Y_{0}$ is $-\frac{s}{2 d}$. We describe the localization contribution following [11]:

Edge terms: Here we have the weights of the $H^{1}$ 's at the numerator, and of the $H^{0}$ 's at the denominator. A basis of $H^{1}\left(C, f^{*} \mathcal{O}(-1 / 2)\right)=$ $H^{1}\left(C, \mathcal{O}\left(-\frac{d}{2}\right)\right)$ is given by $Y_{0}^{-2(i+1)}$ for $0 \leq i \leq \frac{d-3}{2}$. With our choice of lifting of weights we obtain:

$$
\frac{s^{\frac{d-1}{2}}}{(2 d)^{\frac{d-1}{2}}}(d-1) ! !
$$


Similarly $H^{1}\left(C, f^{*} \mathcal{O}(-1)\right)=H^{1}(C, \mathcal{O}(-d))$ is generated by $Y_{0}^{-2 i}$ for $1 \leq i \leq d-1$. The contribution is:

$$
\frac{s^{d-1}}{(2 d)^{d-1}}(2 d-2) !
$$

At the denominator $H^{0}\left(C, f^{*} \mathcal{T}_{\mathbb{P}}\right)$ is generated by $Y_{0}^{2 i+1}$, for $0 \leq i \leq$ $\frac{3 d-1}{2}$. The product of weights is:

$$
-\frac{s^{\frac{3 d-1}{2}}}{(2 d)^{\frac{3 d-1}{2}}}(2 d) ! !(d-1) ! !
$$

Vertex terms: Pulling back the bundles to the contracted component, we obtain a trivial bundle on the contracted curve. But the action of $\mathbb{Z}_{2}$ on the fiber of the bundle $f^{*} \mathcal{O}(-1)$ is trivial, whereas on the other two bundles it is not. After pull-pushing, we obtain two copies of the dual of the Hodge bundle on the genus $g$ hyperelliptic cover corresponding to the map $f_{\mid E}$, linearized with the appropriate weights. The contribution is therefore the equivariant euler class of

$$
e^{e q}\left(\mathbb{E}^{\vee}\left(\frac{1}{2}\right) \oplus \mathbb{E}^{\vee}\left(-\frac{1}{2}\right)\right)=\left(\frac{s}{2}\right)^{2 g}
$$

where the equality follows from Mumford's relation ([13]).

Flag terms: There is no flag contribution since we are pulling back the non-trivial line bundle $L$ on $B \mathbb{Z}_{2}$ via a map with a twisted point.

Automorphisms and node smoothing: For the automorphism of the curve we have a term corresponding to moving the vertex over $\infty$. Together with the node smoothing contribution we obtain:

$$
-\frac{s}{d}\left(\frac{s}{2 d}-\frac{\psi}{2}\right)^{-1}
$$

Putting everything together we obtain:

$$
I_{d, 2 g+1}=(-1)^{g+\frac{d-1}{2}}\left(\frac{d}{2}\right)^{2 g-1} \int_{A d m_{g \rightarrow d_{0,\left(t_{1}, \ldots, t_{2 g+2}\right)}}} \psi^{2 g-1}
$$


The integral is just the hyperelliptic Hurwitz number with value $\frac{1}{2}$ for any $g$, and hence we have:

$$
\mathcal{I}_{d}=\sum_{g \geq 0} I_{d, 2 g+1} \frac{z_{2}^{2 g+1}}{(2 g+1) !}=(-1)^{\frac{d-1}{2}} \frac{2}{d^{3}} \sin \left(\frac{d z_{2}}{2}\right) .
$$

Remark. The $g=0$ term in formula (2.2) corresponds to the invariant with only one stacky insertion, and therefore no contracted curve $F$.

\subsection{Even degree}

In this case, $F$ and $C$ are connected by a non-stacky node, and $C$ is a smooth rational curve. If we choose local coordinates $Z_{0}$ and $Z_{1}$ the map on $C$ is given by $f^{*} X_{0}=Z_{0}^{\frac{d}{2}}$.

\section{Edge terms:}

$$
\frac{s^{\frac{d-1}{2}}}{(2 d)^{\frac{d-1}{2}}}(d-1) ! ! \frac{s^{d-1}}{d^{d-1}}(d-1) ! \frac{(2 d)^{\frac{d}{2}} d^{d}}{2 d ! d ! ! s^{\frac{3 d}{2}}} .
$$

\section{Vertex terms:}

$$
e^{e q}\left(\mathbb{E}^{\vee}\left(\frac{1}{2}\right) \oplus \mathbb{E}^{\vee}\left(-\frac{1}{2}\right)\right)=\left(\frac{s}{2}\right)^{2 g}
$$

Flag terms: Pulling back the non-trivial line bundle $L$ on $B \mathbb{Z}_{2}$ via a map with a regular point, we have the flag term $\frac{s}{2}$.

\section{Automorphisms and node smoothing:}

$$
-\frac{s}{d}\left(\frac{s}{d}-\psi\right)^{-1}
$$

Since the node is not stacky, we also have an additional gluing factor of 2. Including the invariant with no insertions (corresponding to $g=-1$ ), we obtain:

$$
\mathcal{I}_{d}=\sum_{g=-1}^{\infty} I_{d, 2 g+2} \frac{z_{2}^{2 g+2}}{(2 g+s) !}=(-1)^{\frac{d}{2}} \frac{2}{d^{3}} \cos \left(\frac{d z_{2}}{2}\right) .
$$

\subsection{The potential}

Collecting the information from Sections 2.2, 2.4, 2.5 and using the divisor equation we obtain the following Theorem. 
Theorem 2.1. The equivariant genus 0 Gromov-Witten potential of $\mathcal{Z}$ is

$$
\begin{gathered}
F^{\mathcal{Z}}=\frac{z_{0}^{3}}{18 t_{1} t_{2}}-\frac{1}{3} z_{0} z_{1}^{2}+\frac{1}{4} z_{0} z_{2}^{2}-\frac{t_{1}}{4} z_{1} z_{2}^{2}-\frac{1\left(t_{1}+2 t_{2}\right)}{9} z_{1}^{3}-\left(t_{1}+t_{2}\right) G \\
+\left(t_{1}+t_{2}\right)\left(\sum_{d \text { odd }}(-1)^{\frac{d-1}{2}} \frac{2}{d^{3}} \sin \left(\frac{d z_{2}}{2}\right) \mathrm{e}^{d z_{1}} q^{d}\right. \\
\left.+\sum_{d \text { even }}(-1)^{\frac{d}{2}} \frac{2}{d^{3}}\left(\cos \left(\frac{d z_{2}}{2}\right)\right) \mathrm{e}^{d z_{1}} q^{d}\right) .
\end{gathered}
$$

where the first non-zero coefficient of $G$ is in degree 4 and $G^{\prime \prime \prime}=\frac{1}{2} \tan \left(\frac{z_{2}}{2}\right)$.

\section{Checking the CPRC}

Theorem 3.1. The change of variables:

$$
y_{0}=z_{0}, \quad y_{1}=\mathrm{i} z_{2}, \quad y_{2}=z_{1}-\frac{\mathrm{i}}{2} z_{2} \quad q_{1}=-\mathrm{e}^{\mathrm{i} u}, \quad q_{2}=\mathrm{i} q
$$

verifies the $C R C$ for the pair $\mathcal{Z}, Y$.

Proof. A straightforward algebraic substitution checks that (3.1) matches the degree 0 part of the potential of $Y$ with the degree 0 three pointed invariants contribution to the potential of $\mathcal{Z}$, up to a residual contribution of

$$
-\frac{\mathrm{i}}{12}\left(t_{1}+t_{2}\right) z_{2}^{3}
$$

Next we focus on the term:

$$
\left(t_{1}+t_{2}\right) \sum_{d=1}^{\infty} \frac{1}{d^{3}}\left(e^{y_{1}} q_{2}\right)^{d} .
$$

This term appears in the Gromov-Witten potential for $\left[\mathbb{C}^{2} / \mathbb{Z}_{2}\right]$; the change of variables (3.1) on this term coincides with the change of variables for the case of $\left[\mathbb{C}^{2} / \mathbb{Z}_{2}\right]$. Bryan et al. prove $[3$, Corollary 3.4$]$ that after the change of variables (and analytic continuation) one obtains:

$$
-\left(t_{1}+t_{2}\right) G+\frac{\mathrm{i}}{12}\left(t_{1}+t_{2}\right) z_{2}^{3} .
$$

This clears up term (3.2) and completes matching the degree 0 invariants of $\mathcal{Z}$. 
Plugging (3.1) into the remaining two summations we obtain:

$$
\sum_{d=1}^{\infty} \frac{\left(q e^{z_{1}}\right)^{d}}{d^{3}} \mathrm{e}^{\frac{i d u}{2}}(i)^{d}\left[\frac{\mathrm{e}^{-\mathrm{i} \frac{d z_{2}+d u}{2}}+(-1)^{d} \mathrm{e}^{\mathrm{i} \frac{d z_{2}+d u}{2}}}{2}\right] .
$$

Hence for $d$ odd, the expression in the brackets above becomes

$$
(-1)^{\frac{d-1}{2}}\left[\frac{\mathrm{e}^{\mathrm{i} \frac{d z_{2}+d u}{2}}-\mathrm{e}^{-\mathrm{i} \frac{d z_{2}+d u}{2}}}{2 i}\right]=(-1)^{\frac{d-1}{2}} \sin \left(\frac{d\left(z_{2}+u\right)}{2}\right)
$$

and for $d$ even:

$$
(-1)^{\frac{d}{2}}\left[\frac{\mathrm{e}^{\mathrm{i} \frac{d z_{2}+d u}{2}}+\mathrm{e}^{-\mathrm{i} \frac{d z_{2}+d u}{2}}}{2}\right]=(-1)^{\frac{d}{2}} \cos \left(\frac{d\left(z_{2}+u\right)}{2}\right) .
$$

This concludes the proof of Theorem 3.1.

One can obtain the change of variables between $\widehat{F}^{\mathcal{Z}}$ to $\widehat{F}^{X}$ by inverting (3.1) and composing with the change of variables (1.2) between the extended Potentials of $\mathcal{X}$ and $Y$. This proves our main theorem.

Theorem 3.2. The change of variables:

$$
\begin{array}{rlrl}
z_{0} & =x_{0}, \\
z_{1} & =\frac{i}{2 \sqrt{3}}\left((\bar{\omega}-1) x_{1}+(\omega-1) x_{2}\right), & & q=-i \omega \mathrm{e}^{\frac{i}{\sqrt{3}}\left(\bar{\omega} s_{1}+\omega s_{2}\right)}, \\
z_{2} & =\frac{1}{\sqrt{3}}\left(\omega x_{1}+\bar{\omega} x_{2}\right), & & u=-\frac{\pi}{3}+\frac{1}{\sqrt{3}}\left(\omega s_{1}+\bar{\omega} s_{2}\right) .
\end{array}
$$

verifies the $C P R C$ for the pair $\mathcal{X}$ and $\mathcal{Z}$

Remark. These change of variables is not unique, as it depends on a choice for a branch of the logarithm when inverting (3.1). However, no choice will give $u=0$ when $s_{1}, s_{2}=0$, which suggests that the extended potential is not only a natural, but a necessary choice in order to obtain a CPRC statement.

\section{Acknowledgments}

The authors would like to thank Arend Bayer, Aaron Bertram, Jim Bryan, Charles Cadman, Y.-P. Lee, Paul Johnson and Rahul Pandharipande for helpful conversations. They wish to acknowledge MSRI, where this work was concluded during the program in Algebraic Geometry (2009). 


\section{References}

[1] A. Bertram, R. Cavalieri and G. Todorov, Evaluating tautological classes using only Hurwitz numbers, Trans. Amer. Math. Soc. 360(11) (2008), 6103-6111

[2] J. Bryan, T. Graber and R. Pandharipande, The orbifold quantum cohomology of $\mathbb{C}^{2} / \mathbb{Z}_{3}$ and Hurwitz-Hodge integrals, J. Algebraic Geom. $\mathbf{1 7}(1)$ (2008), 1-28.

[3] J. Bryan, T. Graber and R. Pandharipande, The orbifold quantum cohomology of $\mathbb{C}^{2} / Z_{3}$ and Hurwitz-Hodge integrals, J. Algebr. Geom. 17(1) (2008), 1-28

[4] R. Cavalieri, Hodge-type integrals on moduli spaces of admissible covers, in 'The interaction of finite type and Gromov-Witten invariants (BIRS 2003)', eds. D. Auckly and J. Bryan (Geometry and Topology Monographs 8), 2006.

[5] R. Cavalieri, Generating functions for Hurwitz-Hodge integrals, Adv. Math. 218(5) (2008), 1419-1429

[6] T. Coates, A. Corti, H. Iritani and H.-H. Tseng. Computing genuszero twisted [Gromov-Witten] invariants, Duke Math. J. 147(3) (2009), $377-438$.

[7] T. Coates, H.I. Iritani and H.-H. Tseng, Wall-crossings in toric Gromov-Witten theory. I. Crepant examples, Geom. Topol. 13(5) (2009), 2675-2744.

[8] T. Coates and Y. Ruan, Quantum cohomology and crepant resolutions: a conjecture, arXiv:0710.5901.

[9] C. Faber and R. Pandharipande, Logarithmic series and Hodge integrals in the tautological ring, Michigan Math. J. 48 (2000), 215-252 With an appendix by Don Zagier, Dedicated to William Fulton on the occasion of his 60th Birthday.

[10] T. Graber and R. Pandharipande, Localization of virtual classes, Invent. Math. 135(2) (1999), 487-518

[11] K. Hori, S. Katz, A. Klemm, R. Pandharipande, R. Thomas, C. Vafa, R. Vakil and E. Zaslow, Mirror symmetry (Clay Mathematics Monographs 1). American Mathematical Society, Providence, RI, 2003. With a preface by Vafa. 
[12] G. Kerr, Weighted blowups and mirror symmetry for toric surfaces, Adv. Math. 219(1) (2008), 199-250

[13] D. Mumford, Towards an enumerative geometry of the moduli space of curves. In 'Arithmetic and geometry', Vol. II (Progress in Mathematics 36), 271-328. Birkhäuser Boston, Boston, MA, 1983.

[14] Y. Ruan, The cohomology ring of crepant resolutions of orbifolds. In 'Gromov-Witten theory of spin curves and orbifolds' (Contemporary Mathematics 403), 117-126, Amer. Mathematical Society, Providence, RI, 2006.

[15] C. Vafa, String vacua and orbifoldized LG models, Mod. Phys. Lett. A 4(12) (1989), 1169-1185

[16] E. Zaslow, Topological orbifold models and quantum cohomology rings, Comm. Math. Phys. 156(2) (1993), 301-331

Department of Mathematics

Colorado State University

PRINCETON UNIVERSity

NJ, USA

E-mail address: renzo@math.colostate.edu

E-mail address: gtodorov@math.princeton.edu

RECEIVED June 9, 2009 
